\title{
Continuous wave Doppler echocardiography after surgical repair of coarctation of the aorta
}

\author{
KC Chan, D F Dickinson, Gill A Wharton, J L Gibbs
}

\begin{abstract}
Objective-To find how closely pressure gradients across the aortic arch derived from Doppler echocardiography reflect gradients measured by catheter after surgical repair of coarctation of the aorta.
\end{abstract}

Design-Pressure drop across the aortic arch was measured simultaneously by continuous wave Doppler and double lumen catheter in 20 patients with repaired coarctation of the aorta.

Results-The peak pressure drop estimated by Doppler was almost invariably higher than the peak to peak gradient measured by catheter, as might be expected. Wide variation was seen between the Doppler measured pressure drop and instantaneous peak gradient measured by catheter, ranging from +22 to $-17 \mathrm{~mm} \mathrm{Hg}$. The reasons for these differences are unclear but are probably related to a combination of complex flow dynamics in the aortic arch, difficulty in closely aligning the Doppler beam with flow, and inability to measure flow velocity immediately proximal to the site of the surgical repair with continuous wave Doppler.

Conclusions-Continuous wave Doppler echocardiography may significantly overestimate or underestimate the pressure drop after repair of coarctation and it should be interpreted with caution in individual patients. Catheterisation with angiography remains the reference standard for assessment of surgical repair of the aortic arch.

(Br Heart 1992;68:192-4)

Doppler ultrasound has become an established technique for assessment of obstructive lesions with the modified Bernoulli equation (pressure drop $=4 \times$ the square of peak flow velocity). ${ }^{1}$ Pressure gradients derived from Doppler in untreated coarctation of the aorta may be less reliable than similar calculations for valve stenosis, ${ }^{2-4}$ particularly in the presence of an arterial duct ${ }^{5}$ or collateral circulation. ${ }^{6}$ Some doubt has also been cast on the meaning of increased flow velocity across the aortic arch after surgical repair. ${ }^{7}$

Having sometimes noted increased flow velocities in the descending aorta after apparently satisfactory repair of coarctation, we undertook to investigate the accuracy of continuous wave Doppler and the modified
Bernoulli equation in patients who had undergone surgical repair of coarctation.

\section{Patients and methods}

We studied prospectively 20 patients aged between 4.7 and 33 (mean (SD) $15.5(5.3)$ ) years with repaired coarctation of the aorta. Catheterisation was carried out either as part of routine late postoperative review of patch aortoplasty or clinical suspicion of residual or recurrent coarctation. At catheterisation, the ascending and descending aortic pressures were measured simultaneously with a fluid filled, thin walled, French 8 double lumen catheter. Before placing the catheter across the aortic arch simultaneous pressures from the two lumens in the descending aorta were recorded to ensure that identical waveforms were obtained. The pressure waveforms from either side of the aortic arch were recorded at $100 \mathrm{~mm} / \mathrm{s}$ paper speed and were digitised on a graphics pad to compute the peak and peak to peak pressure gradient. A total of five pressure waveforms were digitised and the maximum values taken for comparison with the Doppler derived gradient.

During the recording of the pressures peak flow velocity in the descending aorta was measured with a dedicated continuous wave Doppler transducer positioned in the suprasternal notch. The highest peak flow velocity was recorded and the modified Bernoulli equation (four times the square of the peak velocity) was used to calculate the peak pressure drop for comparison with the catheter measurements. The agreement between the catheter and Doppler derived gradients was compared by the method of Bland and Altman. $^{8}$

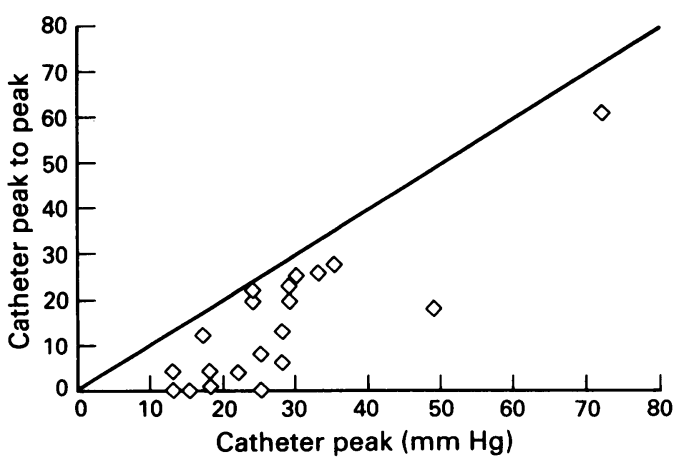

Figure 1 Scatter plot (with line of identity shown) illustrating the differences between peak and peak to peak pressure drop measured by double lumen catheter. pressure drop measured by double lumen catheter. peak measurements, but the difference between the two measurements varies considerably in individual patients. \\ Pepartment of \\ Killingbeck Hospital \\ KC Chan \\ 10 January 1992
}


Figure 2 Comparisons of Doppler estimated peak pressure drop and peak to peak gradient measured by catheter. (A) Scatter plot suggests a similar relation between the two

measurements to that

shown in fig 1. Line of

identity as in fig 1 . ( $B$

Data expressed by the

method of Bland and

Altman. Mean of the two measurements is plotted against the difference between Doppler estimated gradient and catheter peak to peak gradient. Doppler gradient was on average $11.6 \mathrm{~mm} \mathrm{Hg}(8.7)$ higher than catheter peak to peak gradient (range of difference between the two measurements +2 s to $-3 \mathrm{~mm} \mathbf{H g}$ ).
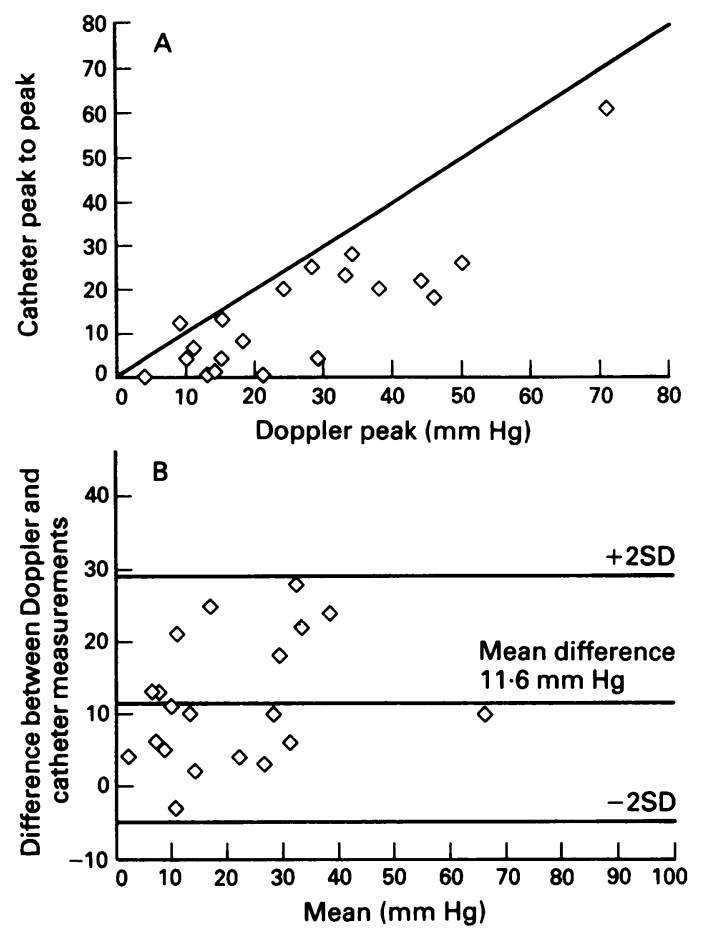

\section{Results}

The figures illustrate the relations between the two gradients measured by catheter and between Doppler and catheter derived measurements. As might be expected, instantaneous peak catheter gradient was invariably higher (mean (SD) $12.6(7.6)$, range 2 to $31 \mathrm{~mm} \mathrm{Hg}$ ) than catheter measured peak to peak gradient, although the difference between the two measurements was inconsistent (fig 1). A similar, also inconsistent, relation was seen between Doppler peak and catheter peak to peak gradients, the mean difference between the two being $11.6(8 \cdot 7)$ and the range +28 to $-3 \mathrm{~mm} \mathrm{Hg}$ (fig 2).

Much closer overall agreement was seen between the Doppler gradient and the cath-

Figure 3 Comparisons of Doppler measured gradient and catheter peak gradient. (A) Scatter plot (with line of identity shown) suggests a closer overall relation between these two measurements. (B) Data expressed by the method of Bland and Altman. The closer overall agreement between these two measurements (compared with those in fig 1) is confirmed, the average difference between Doppler and catheter values being only 0.91 10) $\mathrm{mm} \mathrm{Hg}$. The data points, however, show that in an individual patient the Doppler may

overestimate the true peak gradient by as much as $22 \mathrm{~mm} \mathrm{Hg}$ or

underestimate it by as much as $17 \mathrm{~mm} \mathrm{Hg}$.
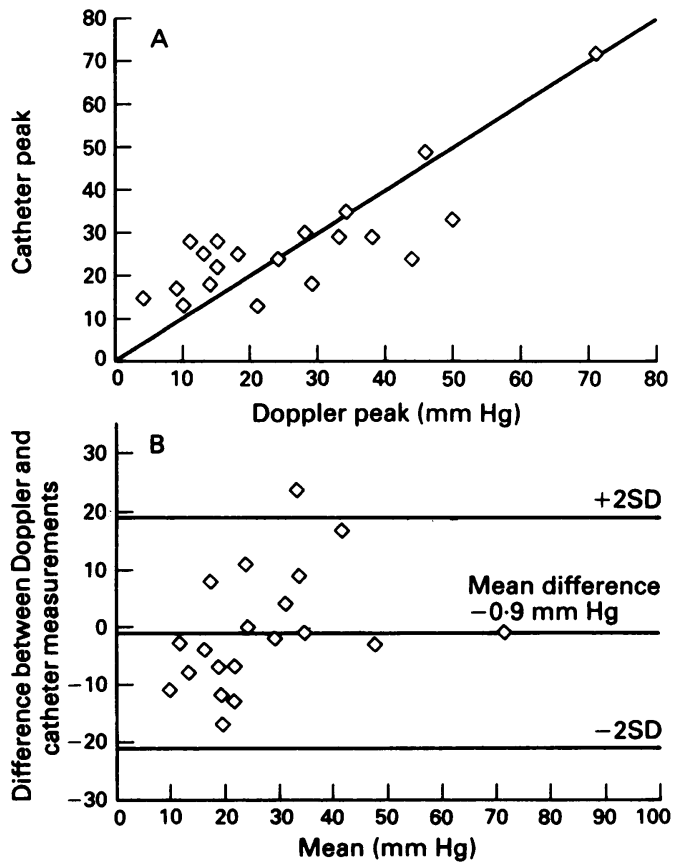

eter instantaneous peak gradient. The mean difference between these two measurements was 0.9 (10) $\mathrm{mm} \mathrm{Hg}$, but the individual differences ranged from +22 to $-17 \mathrm{~mm} \mathrm{Hg}$ (fig 3).

Discussion

The inconsistent relation between instantaneous peak and peak to peak gradient seen in aortic stenosis ${ }^{9}$ appears equally important when assessing pressure gradient across repaired coarctation of the aorta. Disappointingly, however, even when this is taken into account, the use of continuous wave Doppler and the modified Bernoulli equation may still result in substantial overestimation or underestimation of the peak pressure gradient.

The use of a dedicated continuous wave Doppler transducer alone results in difficulty in measuring flow velocity proximal to the site of the aortic repair. In practice this velocity is often less than $1 \mathrm{~m} / \mathrm{s}$ and may be safely ignored in the calculation of pressure drop but if this proximal flow velocity is greater than $1 \mathrm{~m} / \mathrm{s}$, excluding it from the modified Bernoulli equation may result in some overestimation of the pressure gradient. Ideally, therefore, flow velocity in the proximal descending aorta should be measured separately ${ }^{127}$ although close alignment with flow in this part of the arch may be difficult. ${ }^{1}$ Underestimation of pressure drop is most likely to be due to poor alignment of the Doppler beam with the direction of blood flow, a problem that may be minimised but not eliminated by technical expertise during the examination. Further sources of error may lie in the application of the modified Bernoulli equation to potentially complex flow dynamics in an aortic arch distorted by surgical intervention, or scarring, or with long segment stenosis. ${ }^{10}$ Combined measurement of other Doppler values such as diastolic velocity decay ${ }^{4112}$ may enhance detection of important aortic arch obstruction, but even this may be unreliable when extensive collateral circulation has developed. ${ }^{6}$

Previous reports of measurements of nonsimultaneous ultrasound and catheter pressure have suggested that Doppler gradient closely predicts catheter gradient. ${ }^{12}$ Our study of simultaneous catheter and Doppler measurements shows that continuous wave Doppler may considerably underestimate or overestimate aortic arch pressure gradient after surgical repair of the arch and that Doppler ultrasound should be interpreted with caution in this setting. The clinical implication of our findings is that modest increases of flow velocity in the aortic arch after repair of coarctation should not be acted upon without careful consideration of other factors such as the rate of the velocity decay ${ }^{11}$ and the findings on clinical examination at rest and, ideally, during exercise. ${ }^{12}$ Our present practice is to have a low threshold for invasive investigation if the flow velocity in the descending aorta exceeds $3 \mathrm{~m} / \mathrm{s}$ with diminished femoral pulses, with or without upper limb hypertension. 
Aortography remains the reference standard for postoperative assessment of aortic arch repair.

1 Hatle L, Anglesen B. Doppler ultrasound in cardiology: physical principles and clinical applications. Philadelphia: Lea and Febiger, 1985.

2 Marx GR, Allen HD. Accuracy and pitfalls of Doppler evaluation of the pressure gradient in aortic coarctation. evaluation of the pressure gradi Cardiol 1986;7:1379-85.
Am Coln

3 Houston AB, Simpson IA, Pollock JCS, et al. Doppler ultrasound in the assessment of severity of coarctation of ultrasound in the assessment of severity of coarctation of the aorta and int

4 Rao PS, Carey P. Doppler ultrasound in the prediction of pressure gradients across aortic coarctation. Am Heart $J$ 1989;118:299-307.

5 Wilson N, Sutherland GR, Gibbs JL, Dickinson DF, Keeton BR. Limitations of Doppler ultrasound in the diagnosis of neonatal coarctation of the aorta. Int J Cardiol
1989;23:87-9.

6 Scott PJ, Wharton GA, Gibbs JL. Failure of Doppler ultrasound to detect coarctation of the aorta. Int J Cardio 1990;28:379-81.

7 Aldousany A, DiSessa TG, Alpert B, et al. The significance of the Doppler derived gradient across a residual aortic coarctation. Pediatr Cardiol 1990;11:8-14.

8 Bland JM, Altman DG. Statistical methods for assessing agreement between two methods of clinical measurement. Lancet 1986;i:307-10.

9 Currie PJ, Hagler DJ, Seward JB, et al. Instantaneous pressure gradient: A simultaneous Doppler and dual catheter correlative study. J Am Coll Cardiol 1986;7: 800-6.

10 Teirstein PS, Yock PG, Popp RL. The accuracy of Dopple ultrasound measurement of pressure gradients across irregular, dual, and tunnel-like obstructions to blood flow. Circulation 1985;72:577-84

11 Carvalho JS, Redington AN, Shinebourne EA, et al. Continuous wave Doppler echocardiography and coarctation of the aorta: gradients and flow patterns in the assessment of severity. Br Heart J 1990;64:133-7.

12 Gibbs JL. Ultrasound and coarctation of the aorta. $\mathrm{Br}$ Hear $J$ 1990;64:109-10. 